\title{
Advantages of Semiconductor Device Simulator Combining Electromagnetic and Electron Transport Models*
}

\author{
S. M. SOHEL IMTIAZ, SAMIR M. EL-GHAZALY ${ }^{\dagger}$ and ROBERT O. GRONDIN \\ Department of Electrical Engineering, Telecommunications Research Center, Arizona State University, \\ Tempe, AZ 85287-7206
}

(Received 18 May 1997; In final form 10 July 1997)

\begin{abstract}
Physical simulation of semiconductor devices at high frequencies involves not only semiconductor transport issues but also electromagnetic wave propagation issues. In order to obtain the nonlinear and the large-signal characteristics of the semiconductor devices, an electromagnetic model should replace the traditional quasi-static model in the device simulator. In this paper, the advantages of a semiconductor device simulator combining an electromagnetic and an electron transport models are presented. This study is based on a semiconductor device simulator that couples a semiconductor model to the 3D time-domain solution of Maxwell's equations. The electromagnetic wave propagation effects on the millimeter-wave FETs are thoroughly analyzed. The use of the electromagnetic model over the conventional quasi-static model provides the actual device response at high frequencies. It also shows the nonlinear energy build-up along the device width whereas the quasi-static model provides a linear increase of energy. The combined model is capable of predicting the device nonlinearity and harmonic distortion of amplifier circuits at large signal.
\end{abstract}

Keywords: Device simulation, hydrodynamic models, FDTD, full-wave simulators

\section{INTRODUCTION}

With the advancement of semiconductor technology, the techniques required to analyze, design, and optimize the semiconductor devices are becoming increasingly sophisticated. The computer simulation programs are now essential tools for device engineers. These numerical simulations based on physical modeling can be used to predict and provide better understanding of the device behavior. However, the down-sizing of the active device dimensions has presented new challenges to the device and circuit designer. In submicron semiconductor devices, several new transport

* This work is supported by the Army Research Office under contract \# DAAH04-95-1-0252.

${ }^{\dagger}$ Corresponding author. Phone: 602-965-5322, Fax: 602-965-8325, Email: sme@asu.edu. 
phenomena develop and, consequently, have to be considered in device modeling. The electrons do not reach equilibrium transport conditions while traveling along the conducting channel. To incorporate the effects of nonstationary dynamics in semiconductor devices, the hydrodynamic model based on moments of Boltzmann's transport equation is used [1-3]. The transport parameters are taken as functions of average electron energy rather than the local electric field.

The device modeling at high frequencies requires special attention. At high frequencies, the coupling between the electrons and the propagating electromagnetic waves can not be neglected in submicron devices. The short period of the propagating EM wave approaches the electron relaxation time and as the electrons need a finite time to adjust their velocities to the changes in field, electron transport is directly affected by the propagating wave. In such cases, the quasi-static semiconductor device models fail to represent accurately the exact device response. In addition, the electrodes extending along the device width behave like transmission lines with nonlinear characteristics. These facts call for the necessity of incorporating wave effects in a three-dimensional model. This goal can be achieved by taking full account of the varying fields inside the device. The acceptable method for representing these various forces is to combine an electromagnetic model with a semiconductor device model which leads to the Combined Electromagnetic and Solid-State (CESS) simulator [4].

On the other hand, the increasing demand of processing and transmitting more informations at a faster rate, drives the analog and digital electronic systems to operate at higher clock speeds. At the same time, to curtail the production cost, the manufacturers are more inclined towards heavily densed integrated circuits. In these high density integrated circuits, there are many closely spaced active and passive devices. As a result, there are some detrimental effects on the circuit performance at high frequencies due to crosstalk caused by coupling, surface waves and radiation effects. In such cases, the circuit modeling issue becomes more intensive. The circuit design should be based on advanced global model which takes the electromagnetic wave effects into consideration.

The issues like device-wave interaction, electromagnetic coupling, discontinuity problem, linear and nonlinear behavior of passive and active devices, and EM radiation effects are addressed in the global modeling. The computer memory requirement as well as the simulation time is reduced by using a hybridization approach in global modeling. The amplifier is divided into three regions, preserving the physical characteristics of the amplifier circuit by taking the reflections at the breaking points into consideration. The full-wave analysis of each region is performed individually and coupled to the next stage properly with all the required informations from the preceding stage. This technique enables one to use large space step, and hence, large time step in matching networks.

\section{NUMERICAL MODEL}

The CESS simulator is a physically based model which takes care of nonisothermal transport and nonstationary electron dynamics as well as electromagnetic wave propagation effects. This model couples the hydrodynamic model to a 3D timedomain solution of Maxwell's equations. The hydrodynamic model is based on the moments of the Boltzmann's transport equation obtained by integration over the momentum space. The electromagnetic wave propagation effects can be completely characterized by solving Maxwell's equations. These equations are first-order linearly coupled differential equations relating the field vectors, current densities and charge densities at any point in space at any time. The coupling between the two models is established by using the fields obtained from the solution of Maxwell's equation in the semiconductor model to calculate the current densities inside the device. These current densities are used to update the electric and the magnetic fields using Maxwell's equations 
with an applied high frequency sinusoidal excitation. The initialization is provided by solving the hydrodynamic model for the dc charges and currents in response to a specified dc operating point. In this manner, the coupling between the two models results in the overall high frequency characteristics of the semiconductor devices. The details of the mathematical representation and the coupling procedure can be found in [4]. The finitedifference time-domain scheme is used in semiconductor device discretization.

\section{RESULTS AND DISCUSSION}

The MESFET (Fig. 1) used in this work have the following parameters. Gate-source spacing= $0.4 \mu \mathrm{m}$, gate-drain spacing $=0.56 \mu \mathrm{m}$, gate length $=0.24 \mu \mathrm{m}$, undoped GaAs thickness $=$ $0.3 \mu \mathrm{m}$, active layer thickness $=0.1 \mu \mathrm{m}$, gate width $=250 \mu \mathrm{m}$. In order to validate the CESS simulator, a MODFET structure similar to Shawki et al. [5] is simulated to compare the performances. The transconductances are compared with/without taking the traps into account. They exhibit reasonable agreement with each other in Figure 2.

To demonstrate the electromagnetic-wave propagation effects for MESFET, a sinusoidal excitation of peak $0.1 \mathrm{~V}$ and frequency $80 \mathrm{GHz}$ is applied between the gate and the source electrodes. The excitation is applied as a plane source at $z=0$, as shown in Figure 1. The CESS model is then solved for a few $\mathrm{rf}$ cycles to avoid the effects of the

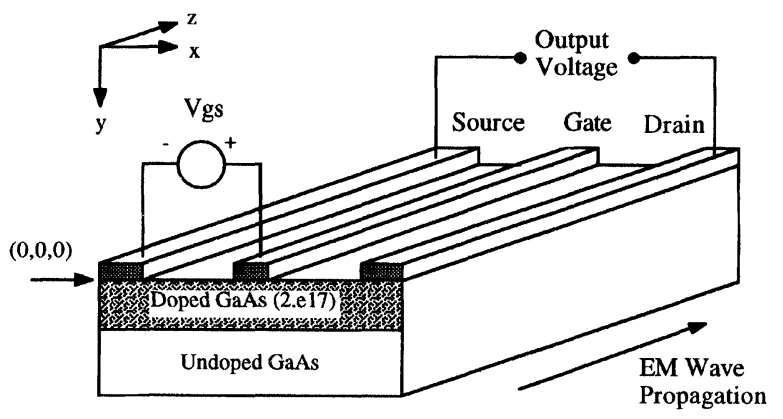

FIGURE 1 The simulated MESFET structure.

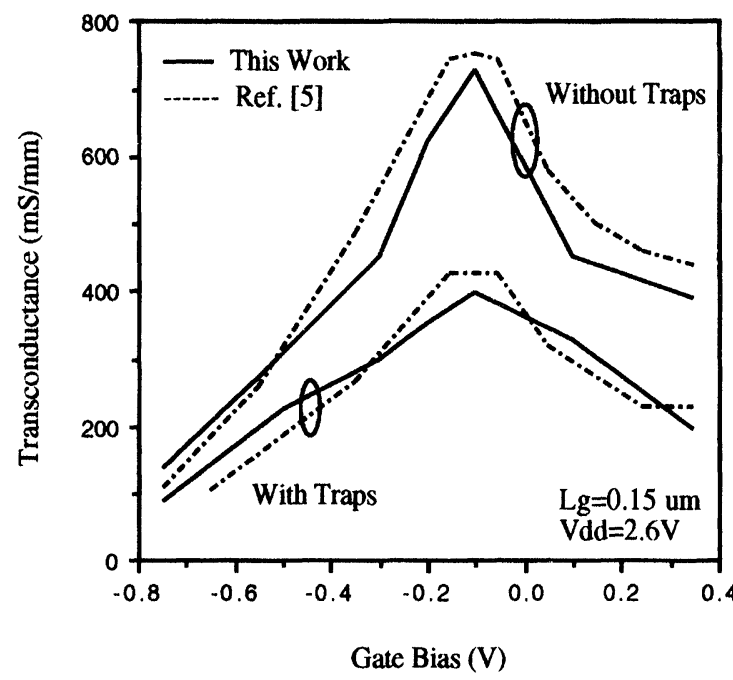

FIGURE 2 Comparison of transconductance of MODFET with Ref. [5] with or without including the traps.

transients on the ac solution. The output is obtained across the drain and the source at several points along the device width in the $z$-direction. The output voltage wave, as shown in Figure 3, first decreases and then increases along the device width. Early in the simulation, the electronic effect is not present and the wave amplitude decreases along the device width. Later, as more and more electromagnetic energy is propagated along the device width, the wave energy builds up, and the wave amplitude increases. This figure clearly

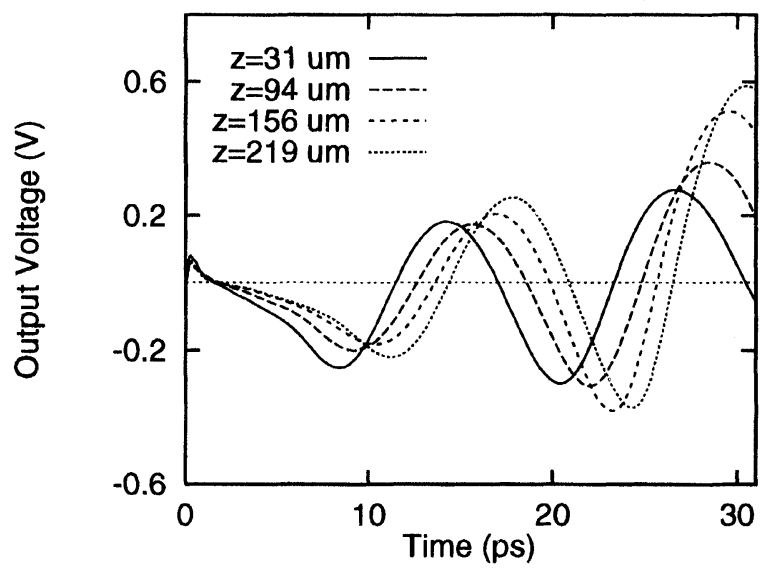

FIGURE 3 The electromagnetic wave propagation effects on output voltages of MESFET for different device widths. 
demonstrates the direct relationship between the device gain characteristics and the electromagnetic wave propagations.

The advantage of using the electromagnetic model in device simulation is demonstrated in Figure 4. MESFET is simulated using the quasistatic model as well as the CESS simulator. In quasi-static model, Poisson's equation is solved to get the electric fields. In electromagnetic model, Maxwell's equations are solved to obtain the electric and the magnetic fields. In Figure 4, the output voltage wave monotonously increases along the device width in quasi-static model. On the other hand, in electromagnetic model, the output voltage wave nonlinearly increases with the device width. This phenomenon is expected due to the device-EM wave interaction. The exchange of energy between the electrons and the electromagnetic wave takes place along the device width. This behavior is absent when the output is obtained from the quasi-static analysis. This figure strongly supports the use of the electromagnetic model for device simulation at high frequencies.

To demonstrate the large signal potential of the CESS simulator, the gain of a $0.5 \mu \mathrm{m} \times 1000 \mu \mathrm{m}$ MESFET is calculated for a small signal of $0.1 \mathrm{~V}$

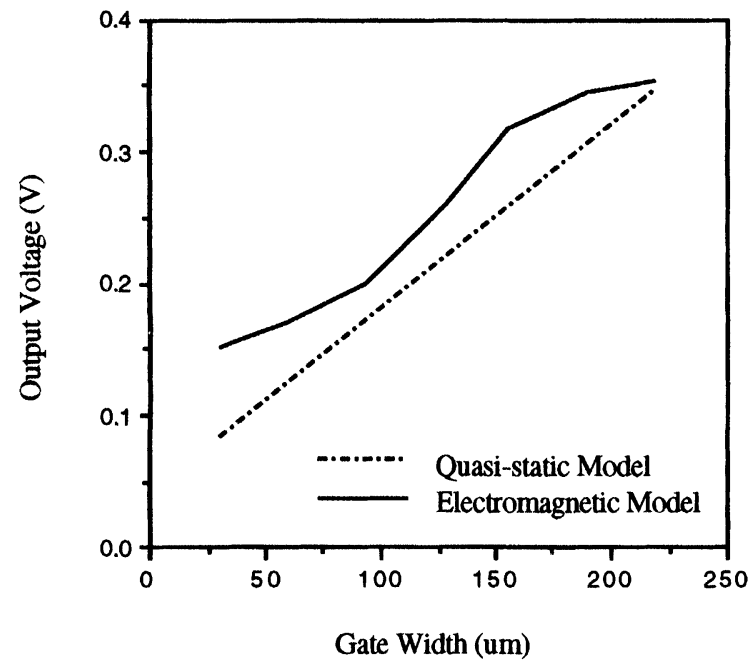

FIGURE 4 The comparison of output voltage variations with device-width obtained from the electromagnetic model and the quasi-static model for MESFET. and a large signal of $0.3 \mathrm{~V}$. As shown in Figure 5, the gain becomes lower as the amplitude increases, which is expected. The strength of this approach is not in simply confirming that larger amplitudes reduce the gain, but in estimating the reduction directly, using the physical model.

The potential of the CESS model is further investigated by simulating an amplifier circuit with input and output matching networks as shown in Figure 6. The physical simulation of the entire amplifier is performed using a global modeling technique [6]. The optimized transistor parameters (in terms of maximum gain) used in the amplifier

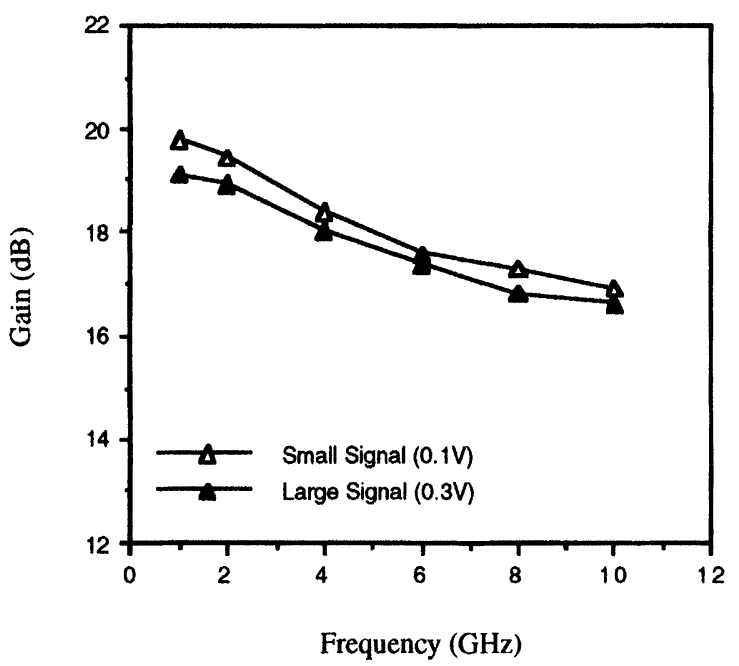

FIGURE 5 The comparison of gain characteristics of MESFET at small and large signal operations.

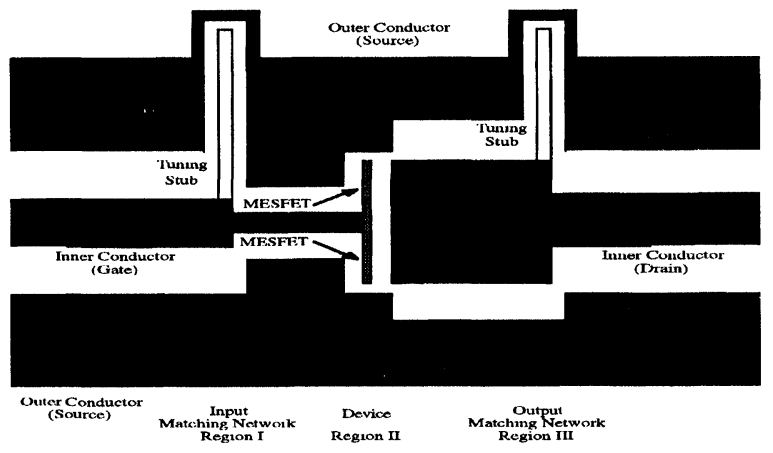

FIGURE 6 GaAs transistor amplifier with input and output matching networks. 
are: gate length $=0.22 \mu \mathrm{m}$, aspect ratio $=2.5$ and the active layer doping $=2.2 \times 10^{17} / \mathrm{cm}^{3}$. The amplifier is designed for $40 \mathrm{GHz}$ frequency. The entire amplifier is simulated by applying a small signal of amplitude $0.1 \mathrm{~V}$ and large signal of amplitudes 0.3 and $1.0 \mathrm{~V}$ at different frequencies. In Figure 7, $S_{21}$ is presented for small and large signals at different frequencies. At the design frequency of $40 \mathrm{GHz}$, the gain is $7.38 \mathrm{~dB}$ for small signal and 6.44 and $5.15 \mathrm{~dB}$ for large signals of 0.3 and $1.0 \mathrm{~V}$, respectively. Thus it is observed that for the same design the gain drops at large signals. In small signal, the gain drops slightly as the frequency is shifted away from the $40 \mathrm{GHz}$ point. On the other hand, in large signal, the gain reduction at frequencies other than design frequency is higher. Nonlinearity in the device behavior is evident from this figure.

Once the large signal response of the amplifier is obtained, it is interesting to study its frequency content and to identify the harmonics. The output wave contains a significant amount of third and fifth harmonic components as shown in Figure 8. For the large signal input of $0.3 \mathrm{~V}$, the output power contains $4.8 \%, 11.6 \%$ and $13 \%$ of the

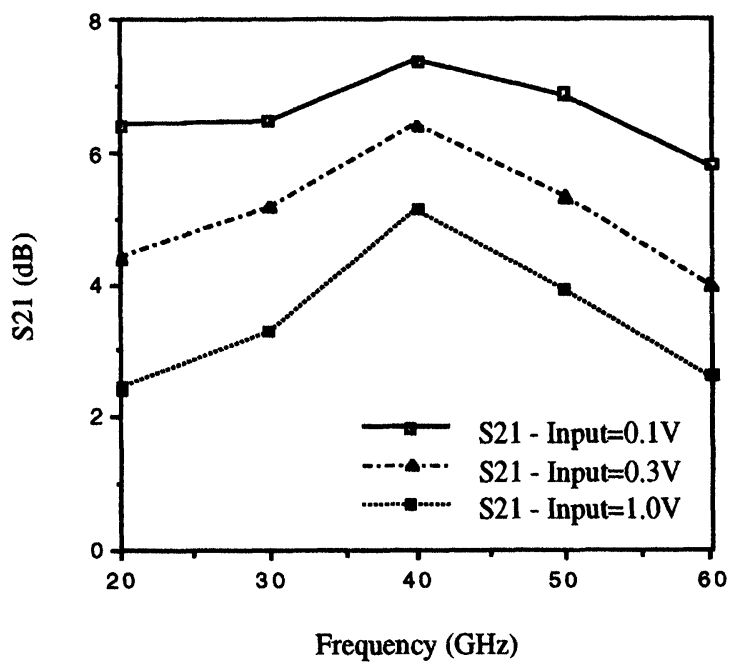

FIGURE 7 The dependence of scattering parameter $S_{21}$ on frequency at small and large signals for the amplifier with the optimized transistor.

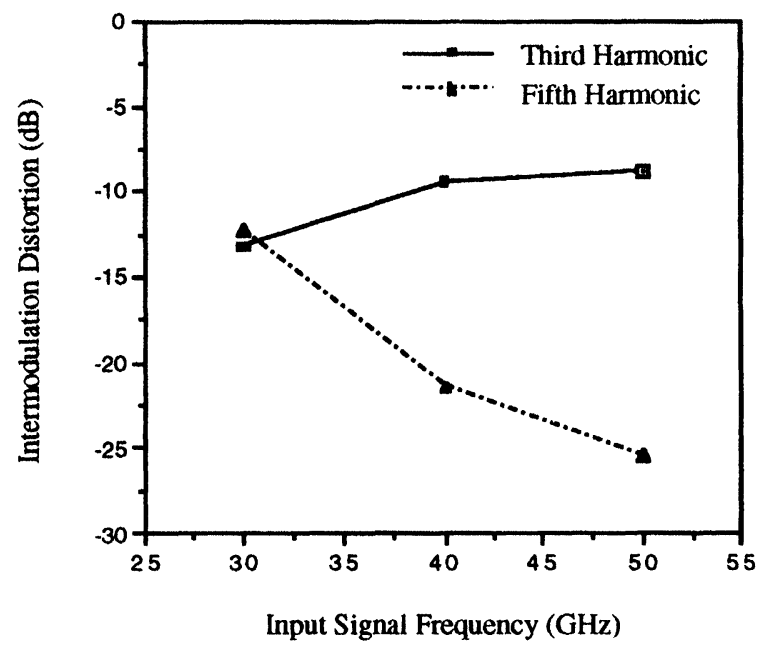

FIGURE 8 The intermodulation distortion at the third and the fifth harmonic components for the large signal of amplitude 0.3 at 30,40 and $50 \mathrm{GHz}$.

fundamental at 30,40 and $50 \mathrm{GHz}$, respectively, at the third harmonic. The fifth harmonic contains $6 \%, 0.7 \%$ and $0.3 \%$ of the fundamental at 30,40 and $50 \mathrm{GHz}$, respectively. This shows that a considerable amount of power is transferred at the harmonic components. Thus the global model is able to predict the nonlinearity of the device behavior and show the different harmonic components generated at the amplifier output due to nonlinearity.

\section{CONCLUSIONS}

Advantages of semiconductor device and circuit simulator combining an electromagnetic and an electron transport models are presented. The simulation confirms that a significant device-wave interaction takes place in semiconductor devices at high frequencies. The use of the electromagnetic model over the conventional quasi-static model provides the actual device response at high frequencies. It also shows the nonlinear energy build-up along the device width whereas the quasistatic model provides a linear increase of energy. An approach towards global modeling of milli- 
meter-wave circuits is also presented in this paper. The global model is able to characterize the electromagnetic coupling, device-EM wave interaction and the EM radiation effects of the very closely spaced integrated circuit amplifier. The global modeling technique is capable of representing the nonlinearity and the harmonic distortion of the amplifier circuit. More effects will be added in the future including thermal and packaging effects. By incorporating all these effects in the circuit simulation, a milestone will be reached towards the comprehensive global modeling.

\section{References}

[1] Carnez, B., Cappy, A., Kaszynski, A., Constant, E. and Salmer, G., "Modeling of a sub-micrometer gate Fieldeffect transistors including effects of nonstationary electron dynamics", J. Appl. Phys., 51(1), 784-790, Jan. 1980.

[2] Snowden, C. M. and Loret, D., "Two-dimensional hotelectron models for short-gate-length GaAs MESFET's", IEEE Trans. Electron Devices, ED-34(2), 212-223, Feb. 1987.

[3] El-Ghazaly, S. M. and Itoh, T., "Two-dimensional numerical simulation of short-gate-length GaAs MESFETs and application to the traveling Gunn domain phenomenon", Int. J. Numerical Modeling, 1, 19-30, Jan. 1988.

[4] Alsunaidi, M. A., Sohel Imtiaz, S. M. and El-Ghazaly, S. M. "Electromagnetic wave effects on microwave transistors using a full-wave time domain model", IEEE Trans. Microwave Theory Tech., 44, 799-808, June 1996.

[5] Shawki, T., Salmer, G. and El-Sayed, O., "MODFET 2-D hydrodynamic energy modeling: optimization of subquarter-micron gate structure", IEEE Trans. Electron Devices, 37, 21 -30, Jan. 1990.

[6] Sohel Imtiaz, S. M., "Physical simulation of high frequency semiconductor devices and amplifier circuits", Ph.D. Dissertation, May 1997.

\section{Authors' Biographies}

S. M. Sohel Imtiaz was born in Dhaka, Bangladesh, in 1966. He received the B.S. and the M.S. degree in electrical engineering in 1988 and 1990, respectively, from Bangladesh University of Engineering and Technology (BUET), Dhaka. He received his Ph.D. degree in electrical engineering from Arizona State University in 1997. Dr. Imtiaz worked as a Lecturer in the department of electrical engineering in BUET from 1989 to 1991. His research interests include modeling, simulation and characterization of microwave semiconductor devices and circuits, device-wave interactions, numerical techniques, and the simulation of microwave amplifiers. He joined Micro Linear Corporation as a Sr. Device Engineer in June 1997.

Samir M. El-Ghazaly received the Ph.D. degree, in Electrical Engineering, from the University of Texas at Austin, Texas, in 1988. He joined Arizona State University as Assistant Professor in August 1988, and became Associate Professor in 1993. Dr. El-Ghazaly is a senior member of IEEE, an elected member of Commissions A and D of URSI, a member of Tau Beta Pi, Sigma Xi and Eta Kappa $\mathrm{Nu}$. He is the secretary of US National Committee of URSI, Commission A. He is also the Chairman of the Chapter Activities Committee of the IEEE Microwave Theory and Techniques Society.

Robert Grondin was born and raised in Michigan. He attended the University of Michigan obtaining the BS, MS and Ph.D. degrees. From 1981 to 1983 he was a post-doctoral research associate at Colorado State university. In 1983 he joined the faculty of the Department of Electrical Engineering at Arizona State University where he is presently an associate professor. He is a senior member of the IEEE and a member of the AAAS. 

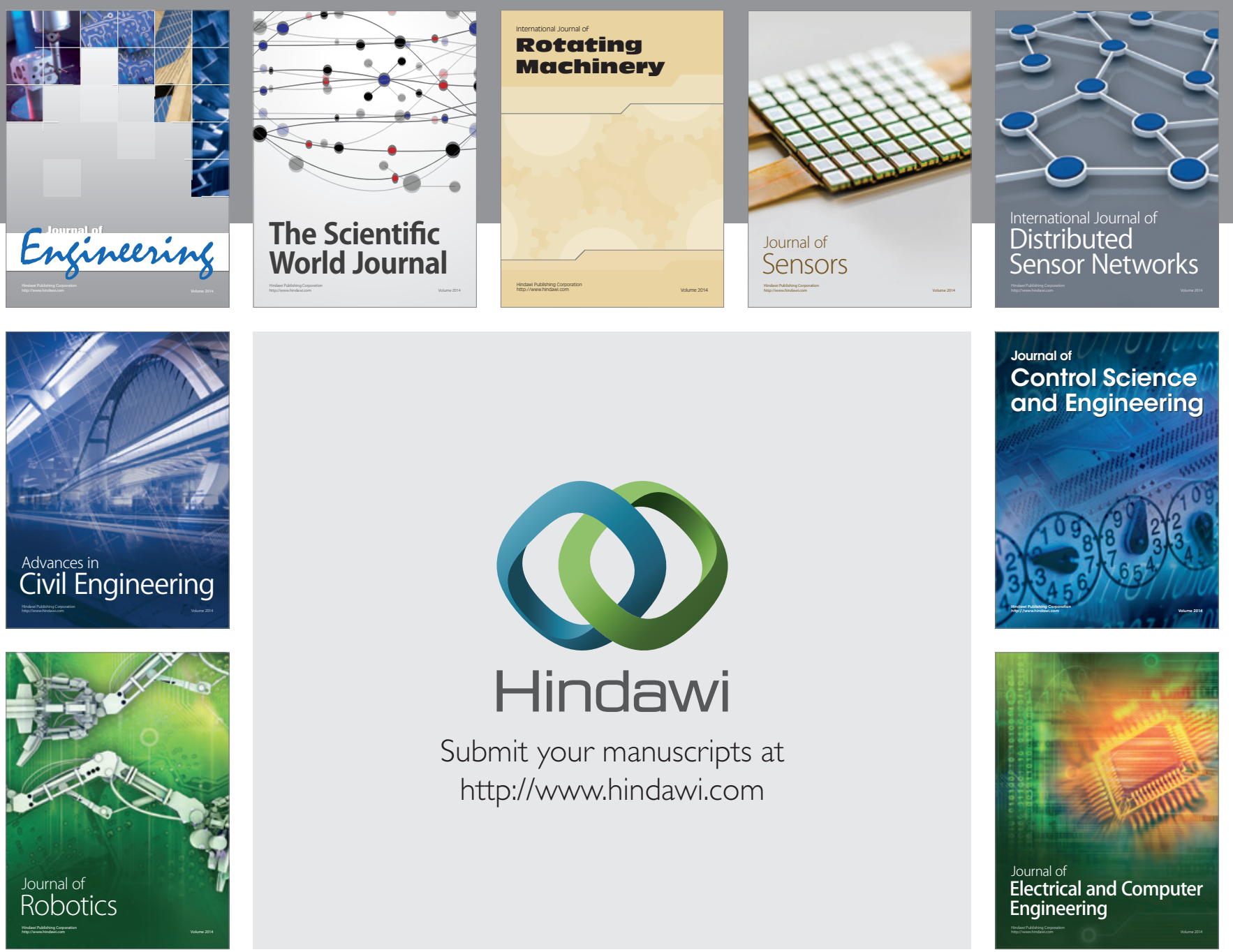

Submit your manuscripts at

http://www.hindawi.com
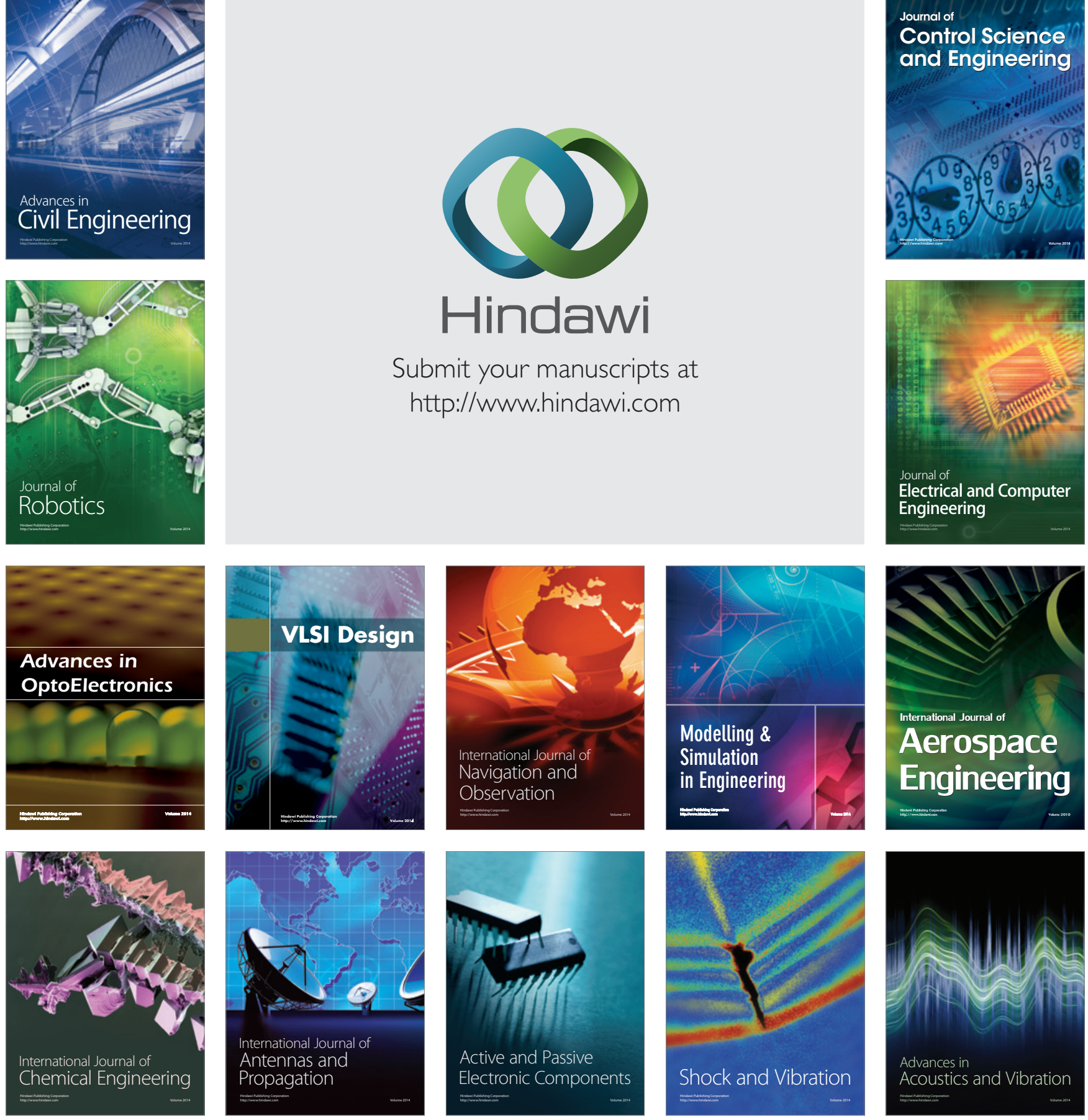